\title{
A CAD Modeling System for the Components Made of Multi Heterogeneous Materials
}

\author{
Feng Zhu and Ke-Zhang Chen \\ Department of Mechanical Engineering, The University of Hong Kong, Hong Kong
}

\begin{abstract}
The heterogeneous materials have been used for satisfying the requirements for special functions of components in various fields. To design and manufacture the components made of multi heterogeneous materials, the computer models for representing them need first to be built so that further analysis, optimization and manufacturing can be implemented based on the models. Since current modeling techniques can capture only the geometric information, a new modeling method has been developed to build the model that can include all the material information along with geometry information. Based on this method, this paper develops a system for modeling the components made of multi heterogeneous materials. With the system, users can apply the functions of current CAD graphic software to build CAD models for their heterogeneous components designed and display both material and geometric information for any cross section of the components they select. As an example for applying the system, the modeling for a special pipe is illustrated.
\end{abstract}

\section{I . INTRODUCTION}

Heterogeneous materials include composite materials, functionally graded materials (FGM), and heterogeneous materials with a periodic microstructure. These heterogeneous materials have been used for satisfying the requirements for special functions of components in various fields, which conventional homogeneous materials cannot meet. A related design method [1,2] for the components made of these heterogeneous materials has been developed under the guidance of Axiomatic Design [3]. To support the new method, the computer models for representing components made of multi heterogeneous materials need first to be built so that further analysis, optimization and manufacturing can be implemented based on the models. However, current modeling techniques can capture only the geometric information [4]. Some researchers [5-9] are focusing on modeling heterogeneous objects by including the variation in constituent composition along with the geometry in the solid model for functionally graded materials. But representing the microstructure of heterogeneous components is beyond their scope [5]. Since the microstructure size is very small, the model consisting of such microstructures has huge number of data to be stored. Even with the help of highspeed modern computers, the processing of the model is extremely difficult and needs extreme care and thoughts for $\mathrm{I} / \mathrm{O}$ operations. Thus, a new modeling method $[10,11]$ has been developed for building the model that can include all the material information (about periodic microstructures, constituent composition, and inclusions) along with geometry information in 3D solid modeling without the problem arising from too much data. Based on this method, this paper develops a software system prototype for modeling the components made of multi heterogeneous materials. With the system, users can apply the functions of current $\mathrm{CAD}$ graphic software to build CAD models for their heterogeneous components designed and display all the information (including both material and geometric information) for any cross section of the components they select. As an example for applying the system, the modeling for a special pipe is illustrated.

\section{II . REVIEW OF THE MODELING METHOD FOR THE COMPONENTS MADE OF MULTI HETEROGENEOUS MATERIALS}

According to this method [10,11], a component made of the three types of materials can be divided into $n$ parts or regions based on their material constituent compositions, and its material constituent composition set can be indicated by:

$C=\left\{C_{i}, i=1,2, \ldots, n\right\}$

According to its material microstructures, the component can also be divided into $m$ regions if there are $m$ different microstructures, and its material microstructure set can be written as:

$S=\left\{S_{j}, j=1,2, \ldots, m\right\}$

Thus, the material region set $(M)$ of the component can be obtained by solving Cartesian product of $C$ 
and $S$ as:

$$
\begin{aligned}
M & =C \times S \\
& =\left\{M_{i j} \mid i \in(1,2,3 \ldots, n), j \in(1,2, \ldots, m)\right\}
\end{aligned}
$$

Each material region has a specified material constituent composition and a specified material microstructure. A CAD model of the component can be formed or integrated by the models of all its material regions. The model for each region consists of three types of sub-models. The first type of submodel is a geometric model that can be made by using current CAD graphic software. That is, the Brep scheme [4] can be used to represent the shape of the whole component and all the borders between different material regions in the component. The last two sub-models are material constituent composition model and material microstructure model, which cannot be represented by $3 \mathrm{D}$ solid model and are in the form of schema [12].

The model for the $i$-th material constituent composition region is designed as the following typical schema with one frame:

$C_{i}=\{$ Coordinate system type: Cartesian, cylindrical, or spherical coordinate system

Origin of coordinate system: $X c_{i}, Y c_{i}, Z c_{i}$

Orientation of coordinate system: $\alpha c_{i}, \beta c_{i}, \gamma c_{i}$

Number of material types: $N c_{i}$

Material types: $A_{1}, A_{2}, \ldots, A_{N C i}$

Material constituent composition function:

$\left[V_{h C i}=f_{h C i}(x, y, z), h=1,2, \ldots, N_{C i}\right.$

[ $\left.\sum_{h=!}^{N c i} V_{h C i}=1,(x, y, z) \in C_{i}\right]$

)

The material microstructure model $(S)$ covers those for composite materials $(R)$, heterogeneous materials with a periodic microstructure $(P)$ and the materials without inclusions and periodic microstructures $(O)$, i.e.,

$S=\left[S_{j}, j=1,2, \ldots, m \mid m=(u+v+w), S_{j} \in(R+P+O)\right]$

where $R=\left[R_{a}, a=1,2, \ldots, u\right], P=\left[P_{b}, b=1,2, \ldots, v\right]$, and $O=\left[O_{d}, d=1,2, \ldots, w\right]$. Since there is no spraying or insertion operation in the region without inclusion and periodic microstructure, there is no need to build a material microstructure model for the region, i.e., $O=\left[O_{d}=\right.$ "nil", $\left.d=1,2, \ldots, w\right]$.

Using the schema theory, the model for the $a$-the material microstructure region with a composite material can be expressed as follows:
$R_{a}=\{$ Coordinate system type: Cartesian, cylindrical, or spherical coordinate system

Origin of coordinate system: $X_{R a}, Y_{R a}, Z_{R a}$ Orientation of coordinate system: $\alpha_{R a}, \beta_{R a}, \gamma_{R a}$ Number of spaying operations: $N_{R a}$

Spraying operation 1:

Spraying material: Code name of material 1

Spraying function: $V_{R a 1}=\left[f_{R a 1}(x, y, z) \mid(x\right.$, $y, z) \in R_{a}$ ]

Spraying operation 2: Spraying material: Code name of material 2 Spraying function: $V_{R a 2}=\left[f_{R a 2}(x, y, z) \mid(x\right.$, $\left.\mathcal{y}, z) \in R_{a}\right]$

Spraying operation $N_{R a}$ : Spraying material: Code name of material $N_{R a}$ Spraying function: $V_{R a N R a}=\left[f_{R a N R a}=(x, y\right.$, z) $\left.\mid(x, y, z) \in R_{a}\right]$

\}

The model for the $b$-th material microstructure region with a heterogeneous material with a periodic microstructure, if its base cell consists of one type of material, can be expressed by a schema as follows:

$P_{b}=\{$ Coordinate system type: Cartesian, cylindrical, or spherical coordinate system

Origin of coordinate system: $X_{P b}, Y_{P b}, Z_{P b}$

Orientation of coordinate system: $\alpha_{P b}, \beta_{P b}, \gamma_{P b}$

Number of insertion operations: 1

Insertion operation 1:

Insertion: Code name of base cell

Insertion material: $\mathrm{Nil}$

Inserting position function: $(x, y, z)=$

$\left[\left(x_{1}\left(t_{1}\right), y_{1}\left(t_{2}\right), z_{1}\left(t_{3}\right)\right) \mid\left(t_{1}, t_{2}, t_{3}\right) \in\right.$ "Integer", $\left.(x, y, z) \in P_{b}\right]$

Dimension: $F_{D 1}(x, y, z)$

Orientation: $F_{\theta 1}(x, y, z)$

Type of RBO: Matrix dominant complex union [13]

\}

\section{ELEMENTS AND WORKFLOW OF THE COMPUTER MODELING SYSTEM}

Based on this modeling method, a CAD modeling system for the components made of multi heterogeneous materials was developed. Its elements and workflow are shown in Fig. 1. The system consists of three main functional modules, that is, 
heterogeneous component modeling module, model processing module, object demonstrating module.

\section{A. Heterogeneous Component Modeling Module}

This main module is used to input all the information needed for modeling and has three submodules. The first sub-module is for 3D geometric modeling using SolidWorks [14], with which the geometric model of each region of a component can be built, stored as a STL file in the computer, and assembled into a complete component. The related geometric parameters will be automatically saved in a geometric parameter record. The second submodule is used to input the related information for material constituent composition modeling in the form of Schema (4). The third sub-module is for material microstructure modeling, with which the related information can be inputted in the form of Schema (6) for composite materials or Schema (7) for heterogeneous material with a periodic microstructure. According to the information inputted, for instance, a name of material type, its ID number and color number will be retrieved from the material type database. The region with a particular material will be rendered into its corresponding color. The region of a single material is illustrated by a single color, while the region of functionally graded material is represented by mixed colors with a gradual change. Likewise, in order to show the difference between composite material and heterogeneous material with a periodic microstructure, special symbols are applied to describe these two regions, as indicated in Fig. 2.

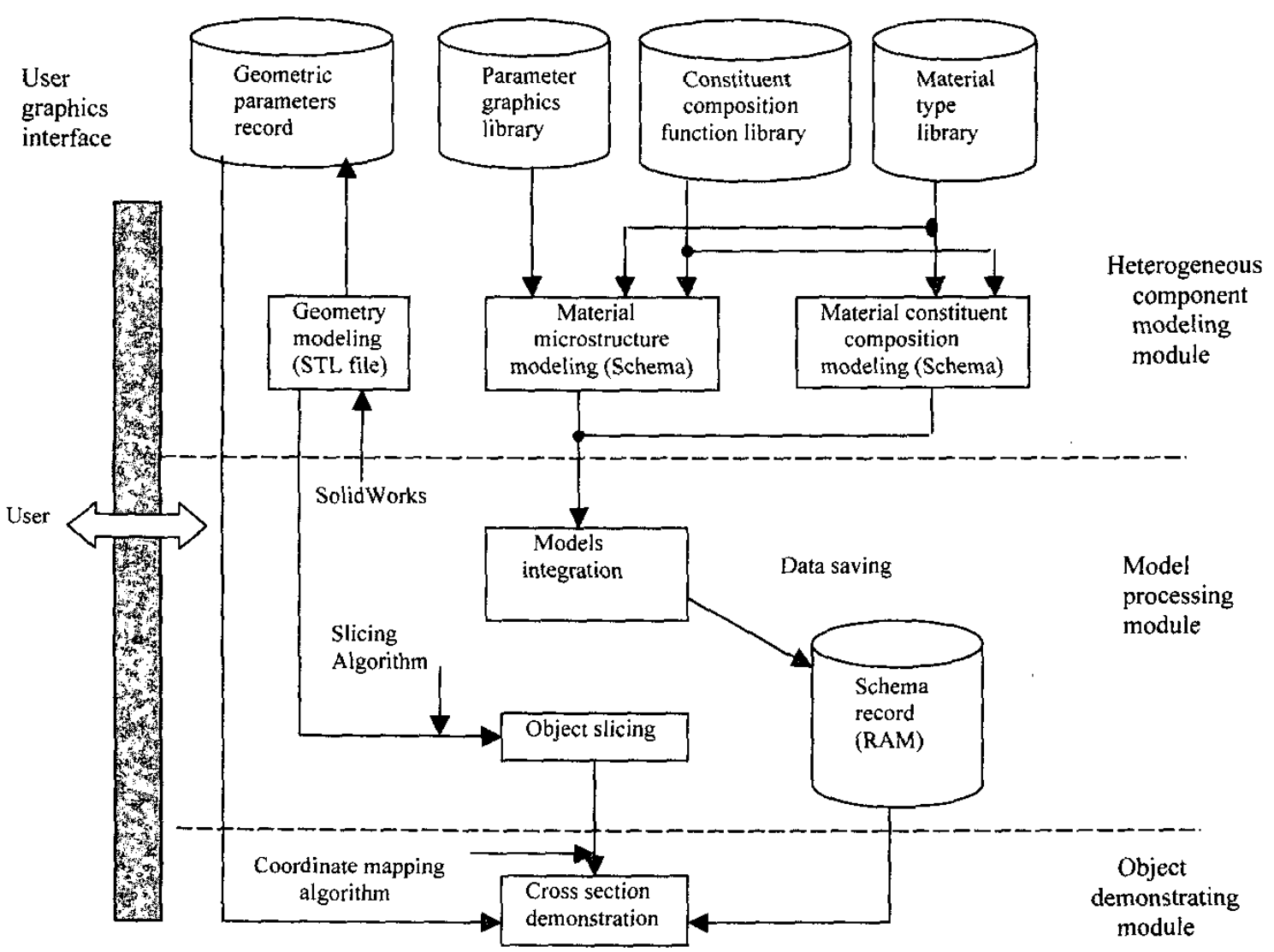

Fig.1 Elements and workflow of the CAD modeling system 


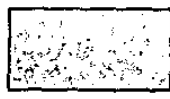

Single material

Functionally graded material

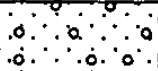

Composite material

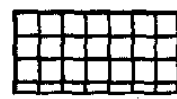

Heterogeneous material with a periodic microstructure

Fig.2 Colors and symbols for representing different heterogeneous material

\section{B. Model Processing Module}

This main module has two functions. The first function is to combine the two types of sub-models of each region, which are inputted in the form of schema, into an integrated model and store the integrated model into a schema record. Thus the integrated model covers all the information of each region for both material constituent composition and microstructure, so that it will be easier and more efficient to deal with the information in subsequent data processing. The second function is to slice the component at a certain position selected by users to obtain the contour of cross section from the geometric parameter record. A slicing algorithm [15] is employed to fulfill it and its procedure goes through (1) reading the STL file, (2) slicing all the related triangles to generate intersectional lines, and (3) sorting out the list of intersectional lines in a head-to-tail fashion.

\section{Object Demonstrating Module}

This main module is to display the cross section with the contours of different material areas and the colors and/or symbols in the different material areas. According to the colors and/or symbol shown in a material area of the cross section, its material type can be easily recognized based on the rules shown in Fig.2. For instance, if a cross hatching symbol appears in a material area, it means that this area consists of a heterogeneous material with a periodic microstructure. If the user wants to know the microstructure in more detail, he or she can select a location with a small rectangle. Once the location for magnification is determined, the pixels within the selected rectangle will be mapped to the world coordinate system, and the information about its microstructure will be found from the schema record, so that the magnified bitmap of correlative microstructure will be displayed in a zoom region according to its dimension, position, direction and magnification ratio.

\section{N. AN EXAMPLE}

As an example for applying the system, the modeling for a special hollow pipe (100 mm long) is illustrated in this section. The internal surface of the pipe is made of ceramic $\left(m_{1}\right)$ for its good temperature properties while its outside part is covered with metal $\left(m_{2}\right)$ due to its good strength. To avoid high stresses at their interface, the middle part is designed with functionally graded materials as shown in Fig.3. All the three parts have a periodic microstructure $\left(M_{1}\right)$ that offers a very small thermal expansion coefficient for the pipe. According to the modeling method

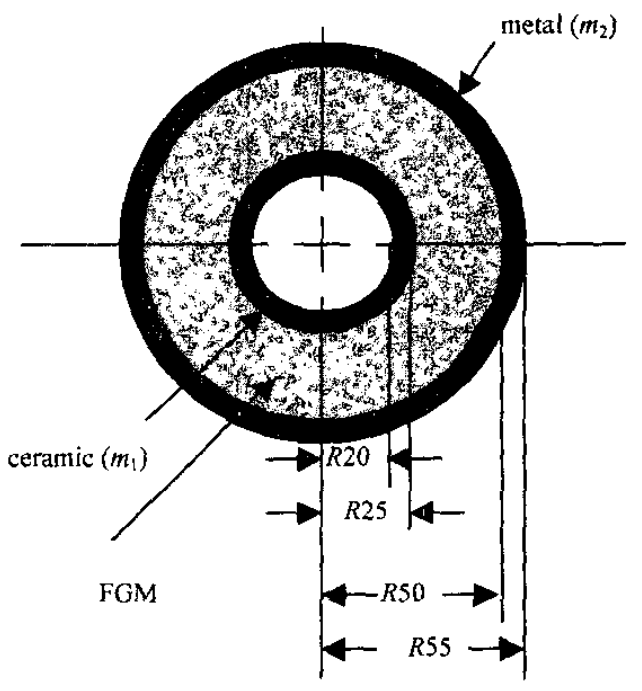

Fig. 3 An example

$[10,11]$, this pipe can be divided into three material constituent composition regions and a material microstructure region, which can be expressed as: 
TABLE I

ATTIBUTES FOR EACH SLOT IN THE MODEL OF MATERIAL CONSTITUENT COMPOSITION REGION

\begin{tabular}{|c|c|c|c|c|c|c|}
\hline $\begin{array}{c}\text { Region No. } \\
\text { of material } \\
\text { constituent } \\
\text { composition }\end{array}$ & $\begin{array}{l}\text { Type of } \\
\text { coordinate } \\
\text { system } \\
\text { of } \\
\text { coordinate } \\
\text { system }\end{array}$ & $\begin{array}{l}\text { Origin } \\
\text { of } \\
\text { coordinate } \\
\text { system }\end{array}$ & $\begin{array}{l}\text { Number } \\
\text { of } \\
\text { materials }\end{array}$ & $\begin{array}{l}\text { Material } \\
\text { types }\end{array}$ & $\begin{array}{c}\text { Material } \\
\text { composition } \\
\text { function }\end{array}$ \\
\hline 1 & $\begin{array}{c}\text { Cylindrical } \\
\text { coordinate } \\
\text { system }\end{array}$ & $0,0,0$ & $0,0,0$ & 1 & $m_{1}$ & even \\
\hline 2 & & & $m_{1}$ & \\
\hline 3
\end{tabular}

$C=\left\{C_{1}, C_{2}, C_{3}\right\}$

$S=\left\{S_{j}, j=1 \mid S_{j} \in(R+P+O)\right\}=\left\{P_{1}\right\}$

The material regions of the component can be thus obtained by solving Cartesian product of $C$ and $S$ as:

$$
\begin{aligned}
M & =C \times S=\left\{M_{11}, M_{21}, M_{31}\right\} \\
& =\left\{\left(C_{1}, P_{1}\right),\left(C_{2}, P_{1}\right),\left(C_{3}, P_{1}\right)\right\}
\end{aligned}
$$

Using the CAD modeling system developed, the $3 \mathrm{D}$ geometric models of the three regions should be first built, saved as STL files (cl.stl, c2.stl, and c3.stl), and finally assembled into a complete pipe. Thus, their geometric parameters, for example, their centers, radii, and lengths are derived by the system and stored in the geometric parameter record. These parameters will be used to specify the attributes of their material constituent composition model and material microstructure model in the procedure of building these two models. The material constituent composition models for the three regions can be built according to Schema (4), where all the attributes for the slots of each model are listed in Table I. The material microstructure models for the three regions are built according to Schema (7) as:

$$
\begin{aligned}
\mathrm{P}_{1}= & \text { Coordinate system type: cylindrical } \\
\text { coordinate system } & \\
\text { Origin of coordinate system: } 0,0,0 & \text { Orientation of coordinate system: } 0,0,0 \\
& \text { Number of insertion operations: } 1 \\
\text { Insertion operation } 1: & \\
& \text { Insertion: P1 } \\
& \text { Insertion material: Nil } \\
& \text { Insertion function: } \\
& \left\{\begin{array}{l}
\rho_{m, n, k}=\frac{D_{o}\left(1-(1+1 \%)^{m+1}\right)}{1-(1+1 \%)} \\
\theta_{m, n, k}=2 \pi n / N(m, n, k=0,1,2 \ldots) \\
z_{m, n, k}=k D_{0}
\end{array}\right.
\end{aligned}
$$

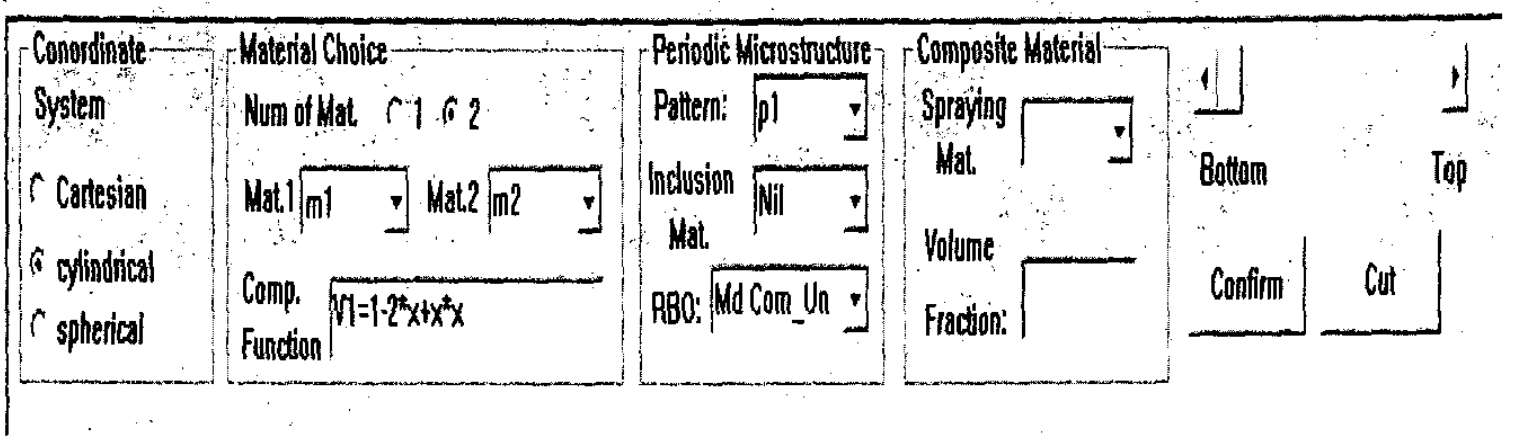

Fig. 4 The control panel 
Dimension:

$D=D_{o}(1+1 \%)^{m} \quad(m=0,1,2 \ldots)$

Orientation:

$\theta=2 \pi n / N \quad(n=0,1,2 \ldots)$

Type of RBO: Matrix dominant complex_union

\}

where $m, n$, and $k$ are the serial numbers of the microstructures in the radial direction ( $\rho)$, circle direction $(\theta)$, and $z$ axis direction respectively, and $N$ denotes the total number of the microstructures of one circle. $D_{o}$ is the dimension of the microstructure at the origin of the local coordinate system. The dimension of each microstructure increases by a fixed ratio from inside to outside in the radial direction and all of the microstructure sizes are the same in the same circle. In the present system, this increase ratio is assumed as $1 \%$, and can be changed according to the design requirements.

Using the interface offered by the system, these schemas can be inputted as shown in fig.4. After these operations, user can open the file and move a slide button on the control panel to select the slicing position. Once the position is confirmed, the cross section selected can be shown with the contours of different material areas and the colors and symbols in the different material areas on the screen of monitor as shown in Fig.5 (a). When the user choose a location for magnification using the mouse, a zoomed image will appear in the zoom work window,

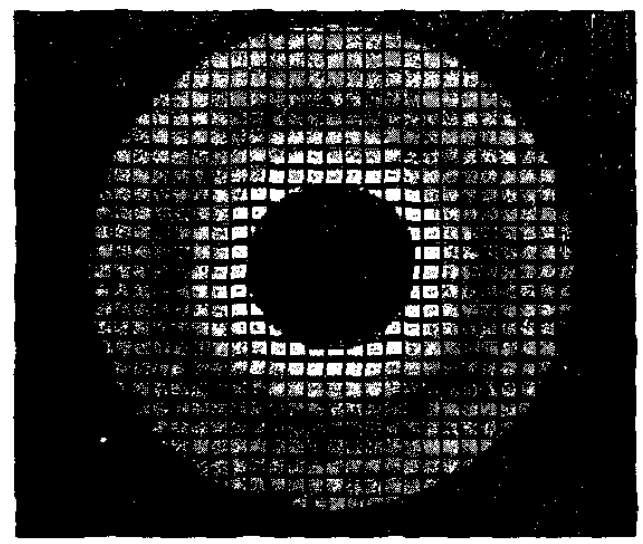

Fig. 5 (a) The cross-section of the pipe as shown in Fig. 5(b), where detailed microstructures can be observed.

\section{CONCLUSIONS}

Based on a new CAD modeling method, this paper develops a system prototype for modeling the components made of multi heterogeneous materials. The system consists of three main functional modules, that is, heterogeneous component modeling module, model processing module, object demonstrating module. The first main module is used to input all the information needed for modeling, including geometric model and different schemas. The second main module can combine all the schemas into an integrated model and slice the component at a certain position selected by users to obtain the contour of cross section from the geometric parameter record. The last main module is used to display the cross section with the contours of different material areas and the colors and/or symbols in the different material areas and to show the detailed microstructures if needed. With the system, users can use the functions of current CAD graphic software to build CAD models for their heterogeneous components designed and display all the information for a cross section of the components they select. The example introduced in the paper has verified the CAD modeling system.

\section{ACNOWLEGEMENT}

The reported research is supported by Competitive

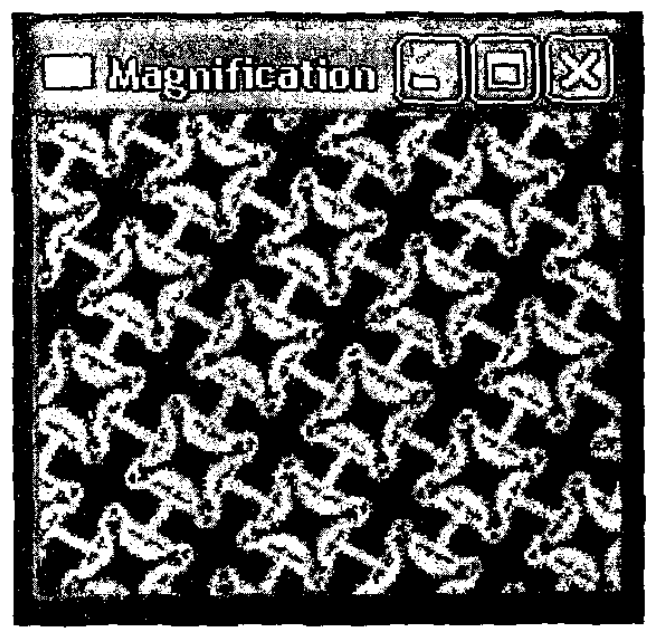

Fig. 5 (b) The magnification of microstructures 
Earmarked Research Grant of Hong Kong Research Grants Council (RGC) under project code: HKU $7062 / 00 \mathrm{E}$. The financial contribution is gratefully acknowledged.

\section{REFERENCES}

[1] K.Z Chen, X.A Feng, "Towards design method for heterogeneous components", in Proceedings of the $13^{\text {th }}$ International Conference on Engineering Design, Glasgow, Scotland, UK, 2001, pp. 445-452.

[2] K.Z Chen, X.A Feng, "Computer-aided design method for the components made of heterogeneous materials", Computer-Aided Design, vol. 35, no. 5, 2003, pp. 453-466.

[3] N.P Suh, The Principle of Design, Oxford University Press, Inc., New York, 1990.

[4] K Lee, Principle of CAD/CAM/CAE System, Addison-Wesley Longman, Inc., Reading, 1999.

[5] V Kumar, D Dutta, "An approach to modeling \& representation of heterogeneous objects", Journal of Mechanical Design, vol.120, 1998, pp. 659-667.

[6] T.R Jackson, H Liu, N.M Patrikalakis, E.M Sachs, M.J Cima, "Modeling and designing functionally graded material components for fabrication with local composition control", Materials and Design, vol. 20(2/3), 1999, pp. 63-75.

[7] Y.K Siu, S.T Tan, "Source-based heterogeneous solid modeling", Computer- Aided Design, vol. 34, no. 1, 2002, pp. 41-55.
[8] Y.K Siu, S.T Tan, "Modeling the material grading and structures of heterogeneous objects for layered manufacturing", Computer-Aided Design, vol.34, no. 10, 2002, pp. 705-716.

[9] S. Morvan, G.M Fadel, "MMA-Rep, A VRepresentation for Multi-material Object", Software Solutions for Rapid Prototyping, PEP Press, UK, 2002.

[10] K.Z Chen, X.A Feng, "A modeling method of heterogeneous components", in Proceedings of International Conference on Manufacturing Automation (ICMA2002), Hong Kong, December, 2002, pp.65-72.

[11] K.Z Chen, X.A Feng, "CAD modeling for the components made of multi heterogeneous materials and smart materials", ComputerAided Design, accepted for publication on April 1, 2003.

[12] D.H Jonassen, K Beissner, M Yacci, Structural knowledge: techniques for representing, conveying, and acquiring structural knowledge, Lawrence Erlbaum Associates, Inc., Hillsdale, New Jersey, 1993.

[13] W Sun, F Lin, X Hu, "Computer-aided design and modeling of composite unit cells", Composite Science and Technology, vol.61, 2001, pp. 289-299.

[14] R Wysack, Designing parts with SolidWorks, CAD/CAM Publishing, San Diego, 1997.

[15] C.F Kirschman, C.C, Jara-Almonte "A parallel slicing algorithm for solid freeform fabrication processes", in Solid Freeform Fabrication Proceedings, 1992, pp 26-33. 